\title{
PSEUDO-INJECTIVE MODULES WHICH ARE NOT QUASI-INJECTIVE
}

\author{
MARK L. TEPLY ${ }^{1}$
}

ABSTRACT. For certain rings with infinitely many nonisomorphic simple left modules, a method is given for constructing pseudo-injective modules which are not quasi-injective. This method is used to produce examples of such modules over a commutative ring.

Let $R$ be a ring with unity. All modules considered here will be unital left $R$-modules. A module $M$ is called quasi-injective (pseudo-injective) if, for every submodule $N$ of $M$, every $R$-homomorphism ( $R$-monomorphism) from $N$ to $M$ can be extended to an $R$-endomorphism of $M$ [5] ([6]). Every quasiinjective module is pseudo-injective. In previous papers (e.g. [4], [6], [7]), most of the results on pseudo-injective modules are of the form, "if $R$ satisfies a suitable hypothesis, then certain pseudo-injective modules are quasiinjective." The intent of most of the work, then, was to show that pseudoinjectives were generally always quasi-injective (e.g. see the comment at the end of the Introduction to [7]). Indeed, the only two examples of pseudoinjective modules which are not quasi-injective have recently appeared in the literature (see [2] and [4]). Both of these modules have precisely five submodules and have Loewy length 2 .

In this note, we give a construction for forming pseudo-injective modules which are not quasi-injective. This construction yields examples which answer in the negative the following two questions of S. K. Jain [3] (see also [4]): (i) Is every pseudo-injective module over a commutative ring quasiinjective? (ii) Is every nonsingular pseudo-injective module quasi-injective? Using an example of Fuchs [1], we can also apply our construction to show that a pseudo-injective module which is not quasi-injective may have arbitrarily large Loewy length.

Received by the editors February 8, 1974 .

AMS (MOS) subject classifications (1970). Primary 16A52; Secondary 13C10.

Key words and phrases. Quasi-injective module, pseudo-injective module, nonsingular module, Loewy length.

${ }^{1}$ The author gratefully acknowledges the financial support of the National Science Foundation under grant GP-39255. 
We now introduce some notation which we will use throughout this note. Let $I$ be an index set, and let $\left\{M_{i}\right\}_{i \in I}$ be a set of modules. For each $i \in I$, let $m_{i} \in M_{i}$. By $\left\langle m_{i}\right\rangle$ we mean the element of $\Pi_{i \in I} M_{i}$ whose $i$ th coordinate is $m_{i}$ for each $i \in I$. By $\left\langle m_{j}\right\rangle^{*}$ we mean the element of $\Pi_{i \in I} M_{i}$ with $m_{j}$ as its $j$ th coordinate and 0 for all other coordinates.

Now we can state the result which is a construction for pseudo-injective modules which are not quasi-injective.

Proposition. Let I be an infinite set, and let $\left\{M_{i}\right\}_{i} \in$ be a set of pseudoinjective $R$-modules each of which has nonzero socle. For each $i \in I$, assume that there exists $r_{i} \in R$ such that

(a) $r_{i} m=m$ for all $m \in M_{i}$, and

(b) $r_{i} m=0$ for all $m \in M_{j}$ with $j \in I-\{i\}$.

For each $i \in I$, let $m_{i} \in \operatorname{Soc} M_{i}$ such that $\left(0: m_{i}\right)$ is a maximal left ideal. Define $M$ to be the R-submodule of $\Pi_{i \in I} M_{i}$ generated by $\bigoplus \Sigma_{i \in I} M_{i}$ and $\left\langle m_{i}\right\rangle$. If $H=\left\{r \in R \mid r \in\left(0: m_{i}\right)\right.$ for all but finitely many $\left.i \in I\right\}$ is a maximal left ide. al of $R$, then the following statements are valid.

(1) $M$ is not quasi-injective.

(2) $M$ is pseudo-injective if and only if the set $S=\{i \in I \mid$ there exists a monomorphism $f: R m_{i} \rightarrow M_{i}$ such that $\left.f\left(m_{i}\right) \neq m_{i}\right\}$ has finite cardinality.

Before proving the proposition, we need two technical lemmas which use the notation of the proposition. $i \in I$.

Lemma 1. If $\left\langle y_{i}\right\rangle \in M-\left(\bigoplus \Sigma_{i} \in I^{M_{i}}\right)$, then $y_{i}=0$ for at most finitely many

Proof. Since $H$ is a maximal left ideal of $R, M / \bigoplus \Sigma_{i \in I} M_{i}$ is a simple left $R$-module. Hence there exist $r \in R$ and $\left\langle d_{i}\right\rangle \in \bigoplus \Sigma_{i \in I} M_{i}$ such that $\left\langle r y_{i}+d_{i}\right\rangle=r\left\langle y_{i}\right\rangle+\left\langle d_{i}\right\rangle=\left\langle m_{i}\right\rangle$. It follows from the choice of the $m_{i}$ that only finitely many of the $y_{i}$ can be 0 .

Lemma 2. Let $f_{i}: R m_{i} \rightarrow M_{i}$ for each $i \in I$. If $f: M \rightarrow M$ is an extension of $\bigoplus \Sigma_{i \in I} f_{i}$, then $f\left(\left\langle m_{i}\right\rangle\right)=\left\langle f_{i}\left(m_{i}\right)\right\rangle$.

Proof. Let $\pi_{i}: M \rightarrow M_{i}$ be the projection map $(i \in I)$. Set $f\left(\left\langle m_{i}\right\rangle\right)=$ $\left\langle k_{i}\right\rangle$. Then

$$
\begin{aligned}
k_{i} & =\pi_{i}\left\langle k_{i}\right\rangle=\pi_{i}\left\langle k_{i}\right\rangle^{*}=\pi_{i}\left(r_{i}\left\langle k_{i}\right\rangle\right)=\pi_{i}\left(r_{i} f\left(\left\langle m_{i}\right\rangle\right)\right) \\
& =\pi_{i} f\left(r_{i}\left\langle m_{i}\right\rangle\right)=\pi_{i} f\left(\left\langle m_{i}\right\rangle^{*}\right)=f_{i}\left(m_{i}\right) .
\end{aligned}
$$

Proof of the proposition. (1) Partition $I$ into two disjoint infinite sets, $J$ and $K$. For each $i \in I$, define $f_{i}: R m_{i} \rightarrow M_{i}$ to be the inclusion map if 
$i \in J$ and the zero map if $i \in K$. If $M$ were quasi-injective, then $\bigoplus \Sigma_{i \in l} f_{i}$ would extend to a homomorphism $f: M-M$. By Lemma 2, $f\left(\left\langle m_{i}\right\rangle\right)=\left\langle f_{i}\left(m_{i}\right)\right\rangle$ $\in M$. Since $f_{i}\left(m_{i}\right)=m_{i} \neq 0$ for $i \in J$ and $f_{i}\left(m_{i}\right)=0$ if $i \in K$, this contradicts Lemma 1 . Hence $M$ is not quasi-injective.

(2) Let $M$ be pseudo-injective. Suppose that the set $S$ has infinite cardinality. Partition $S$ into two disjoint infinite sets $T$ and $S-T$. For each $i \in T$, let $f_{i}: R m_{i} \rightarrow M_{i}$ be a monomorphism such that $f_{i}\left(m_{i}\right) \neq m_{i}$. For each $i \in I-T$, define $f_{i}$ to be the inclusion map $f_{i}: R m_{i} \rightarrow M_{i}$. Since $M$ is pseudo-injective, $\bigoplus \Sigma_{i \in I} f_{i}$ extends to $f: M \rightarrow M$. By Lemma $2, f\left(\left\langle m_{i}\right\rangle\right)=$ $\left\langle f_{i}\left(m_{i}\right)\right\rangle \in M$. Hence

$$
\left\langle m_{i}-f_{i}\left(m_{i}\right)\right\rangle=\left\langle m_{i}\right\rangle-\left\langle f_{i}\left(m_{i}\right)\right\rangle \in M .
$$

By our choice of $f_{i}(i \in I)$, this contradicts the result of Lemma 1 .

Conversely, suppose that $S$ has finite cardinality. Let

$$
M_{0}=\operatorname{ker}\left(\sum_{i \in S} \pi_{i}\right),
$$

where $\pi_{i}: M \rightarrow M_{i}$ is the canonical projection. Then $M=M_{0} \oplus\left(\bigoplus \Sigma_{i} \in S_{i}\right)$. By (a) and (b) any submodule of $M$ is a direct sum of submodules of the $M_{i}$ $(i \in S \cup\{0\})$, and no nonzero submodule of $M_{i}$ is a homomorphic image of a submodule of $M_{j}$ for $i \neq j(i, j \in S \cup\{0\})$. Hence any monomorphism from a submodule of $M$ to $M$ must be a direct sum of monomorphisms from submodules of $M_{i}$ to $M_{i}(i \in S \cup\{0\})$. Since $M_{i}$ is pseudo-injective for each $i \in S$, then $M$ will be pseudo-injective provided that $M_{0}$ is. But it is easy to see that showing $M_{0}$ is pseudo-injective is equivalent to showing $M$ is pseudo-injective whenever $S$ is the empty set. Therefore, we assume $S$ is the empty set and prove that $M$ is pseudo-injective.

Let $N \subseteq M$, and let $g: N \rightarrow M$ be a monomorphism. Let $W=\left\{i \in I \mid\left\langle m_{i}\right\rangle^{*}\right.$ $\notin N\}$. If $0 \neq\left\langle x_{i}\right\rangle \in \Sigma_{i \in W} R\left\langle m_{i}\right\rangle^{*} \cap N$, then for some $j \in W, 0 \neq r_{j}\left\langle x_{i}\right\rangle \in R\left\langle m_{j}\right\rangle^{*} \sim N$. Since $R m_{j}$ is a simple module, there exists $r \in R$ such that $\left\langle m_{j}\right\rangle^{*}=r r_{j}\left\langle x_{i}\right\rangle$ $\in N$, which is a contradiction to our choice of $W$. Hence $\Sigma_{i \in W} R\left\langle m_{i}\right\rangle^{*} \cap N=$ 0 . Similarly, if $0 \neq\left\langle y_{i}\right\rangle \in \Sigma_{i \in W} R\left\langle m_{i}\right\rangle^{*} \cap g(N)$, then for some $j \in W,\left\langle m_{j}\right\rangle^{*} \in g(N)$. From (a) and (b) it follows that $g^{-1}\left(\left\langle m_{j}\right\rangle^{*}\right)=\left\langle k_{j}\right\rangle^{*} \in N$ for some $k_{j} \in M_{j}$. But this allows us to define a monomorphism $g_{j}: R m_{j} \rightarrow M_{j}: r m_{j} \rightarrow r k_{j}$. Since $j \in W$, this forces a contradiction to the assumption that $S$ is the empty set. Hence

$$
\sum_{i \in W} R\left\langle m_{i}\right\rangle^{*} \cap g(N)=0
$$

Therefore we can define a monomorphism 


$$
h:\left(\sum_{i \in W} R\left\langle m_{i}\right\rangle^{*} \oplus N\right) \rightarrow M: \sum s_{i}\left\langle m_{i}\right\rangle^{*}+n \rightarrow \sum s_{i}\left\langle m_{i}\right\rangle^{*}+g(n),
$$

where $s_{i} \in R$ and $n \in N$. For each $i \in I, h$ induces a monomorphism

$$
h_{i}: R m_{i}+\pi_{i} N \rightarrow M_{i}: r m_{i}+\pi_{i}\left\langle n_{j}\right\rangle \rightarrow \pi_{i} h\left(\left\langle r m_{i}\right\rangle^{*}+\left\langle n_{i}\right\rangle^{*}\right),
$$

where $r \in R$ and $\left\langle n_{j}\right\rangle \in N$. Since each $M_{i}(i \in I)$ is pseudo-injective, each $h_{i}$ extends to a homomorphism $f_{i}: M_{i} \rightarrow M_{i}$.

It remains to show that

$$
f: M \rightarrow M:\left\langle a_{i}\right\rangle \rightarrow\left\langle f_{i}\left(a_{i}\right)\right\rangle
$$

defines an extension of $g$. Clearly

$$
f \in \operatorname{Hom}_{R}\left(M, \prod_{i \in I} M_{i}\right), \quad \text { and } \quad f\left(\oplus \sum_{i \in I} M_{i}\right) \subseteq M .
$$

Since $S$ is the empty set, we also have $f_{i}\left(m_{i}\right)=h_{i}\left(m_{i}\right)=m_{i}$; hence $f\left(\left\langle m_{i}\right\rangle\right)$ $=\left\langle m_{i}\right\rangle \in M$. Thus $f \in \operatorname{Hom}_{R}(M, M)$. Finally, if $\left\langle n_{i}\right\rangle \in N$ and $g\left(\left\langle n_{i}\right\rangle\right)=\left\langle y_{i}\right\rangle$, then

$$
\begin{aligned}
y_{j} & =\pi_{j}\left(\left\langle y_{i}\right\rangle\right)=\pi_{j}\left(\left\langle y_{j}\right\rangle^{*}\right)=\pi_{j}\left(r_{j}\left\langle y_{i}\right\rangle\right)=\pi_{j}\left(r_{j} g\left(\left\langle n_{i}\right\rangle\right)\right) \\
& =\pi_{j} g\left(r_{j}\left\langle n_{i}\right\rangle\right)=\pi_{j} h\left(r_{j}\left\langle n_{i}\right\rangle\right)=h_{j} \pi_{j}\left(\left\langle n_{j}\right\rangle^{*}\right)=h_{j}\left(n_{j}\right)=f_{j}\left(n_{j}\right) .
\end{aligned}
$$

Thus $g\left(\left\langle n_{i}\right\rangle\right)=\left\langle y_{i}\right\rangle=\left\langle f_{i}\left(n_{i}\right)\right\rangle=f\left(\left\langle n_{i}\right\rangle\right)$, and hence $f$ extends $g$.

We now use the proposition to construct examples of $R$-modules $M$ which are pseudo-injective, but not quasi-injective. In our first example, $M$ is a torsion module over a commutative integral domain. The reader may wish to compare Example 1 with [4, Theorem 6] and [7, Theorem 2].

Example 1. Let $Z_{2}$ be the field with two elements, and let $R=$ $Z_{2}\left[x_{1}, x_{2}, \ldots\right]$ be the commutative polynomial ring in countably many indeterminants. For each positive integer $i$, let

$$
P_{i}=\left(x_{1}, x_{2}, \cdots, x_{i-1}, 1-x_{i}, x_{i+1}, \cdots\right),
$$

let $M_{i}=R / P_{i}$, let $m_{i}=x_{i}+P_{i}$, and let $r_{i}=x_{i}$. Then $H=\left(x_{1}, x_{2}, \ldots\right)$ is a maximal ideal of $R$; so this situation satisfies the hypothesis of the proposition. Since the additive group of each $M_{i}$ is $Z_{2}$, it follows that the module $M$ constructed in the proposition is pseudo-injective in this case.

In our second example, we see that $M$ can be a nonsingular module over a Boolean ring $R$; indeed, $R$ itself is a pseudo-injective module (i.e. $R$ is a self-pseudo-injective ring).

Example 2. Let $F$ be a finite field, and let $F^{(n)} \cong F$ for each positive integer $n$. Let $R$ be the subring of $\prod_{i=1}^{\infty} F^{(i)}$ generated by $\bigoplus \Sigma_{i=1}^{\infty} F^{(i)}$ and 
$\left\langle 1_{i}\right\rangle$, where $1_{i}$ is the identity element of $F^{(i)}$. For each positive integer $i$, let $M_{i}=R\left\langle 1_{i}\right\rangle^{*}$ and $r_{i}=m_{i}=\left\langle 1_{i}\right\rangle^{*}$. Then $H=\bigoplus \Sigma_{i=1}^{\infty} F^{(i)}$ is a maximal left ideal of $R$. Hence the proposition implies that $M$ is a pseudo-injective module if and only if $F \cong Z_{2}$, the field with two elements. We also note that $M \cong_{R} R$ is a nonsingular module (as $R$ is a von Neumann regular ring).

The modules constructed in the first two examples have Loewy length 2 (see $[1$, p. 174] for the definition). Our last example shows that $M$ can be a (nonsingular) module with arbitrarily large Loewy length.

Example 3. Let $\gamma \geq 2$ be an ordinal, and let $K$ be the field of two elements. In the first part of the proof of [1, Theorem 6], Fuchs constructs a commutative $K$-algebra $L$ (Fuchs calls it $R$ ) such that the Loewy length of $L$ is $\lambda=\gamma+1$. Moreover, every ideal of $L$ contains an idempotent element; so no two distinct modules in the socle of $L$ are isomorphic.

For each positive integer $n$, let $L \cong L^{(n)}$. Define $R$ to be the subring of $\Pi_{n=1}^{\infty} L^{(n)}$ generated by $\bigoplus \Sigma_{n=1}^{\infty} L^{(n)}$ and $\left\langle 1_{n}\right\rangle$, where $1_{n}$ is the identity element of $L_{n}$. Define $M_{i}=L_{\gamma}\left(E\left(R\left\langle 1_{i}\right\rangle^{*}\right)\right)$, where $E\left(R\left\langle 1_{i}\right\rangle^{*}\right)$ is the injective hull of $R\left\langle 1_{i}\right\rangle^{*}$ and $L_{\gamma}$ denotes the $\gamma$ th step in the ascending Loewy series (see $\left[1\right.$, p. 174] for definition). Then $M_{i}$ is an invariant submodule of $E\left(\left\langle 1_{i}\right\rangle^{*}\right)$, and hence $M_{i}$ is quasi-injective. Let $r_{i}=\left\langle 1_{i}\right\rangle^{*}$, and let $m_{i}$ be defined such that $R m_{i}$ is a simple submodule of $M_{i}$. By the structure of $L$, any $R$-monomorphism $R m_{i} \rightarrow M_{i}$ is the inclusion map. Since $H=\bigoplus \Sigma_{n=1}^{\infty} L^{(n)}$ is a maximal ideal of $R$, the proposition implies $M$ is pseudo-injective.

Since any minimal ideal of $L$ is projective, then any minimal ideal of $R$ is also projective and hence nonsingular. Since $M$ is isomorphic to an essential extension of the socle of $R, M$ is nonsingular. Since each $M_{i}$ has Loewy length $\gamma$ and since $R\left\langle m_{i}\right\rangle$ has Loewy length 2 , it follows that $M$ has Loewy length $\gamma$.

Remark. (i) In Example 3, the module $M$ of Loewy length $\gamma \geq 2$ can be made singular by taking

$$
M_{i}=L_{\gamma}\left(E\left(R\left\langle 1_{i}\right\rangle^{*} / \operatorname{Soc} R\left\langle 1_{i}\right\rangle^{*}\right)\right)
$$

and using facts developed by Fuchs [1] to show that any monomorphism $R m_{i}$ $\rightarrow M_{i}$ is the inclusion map.

(ii) Using [1, Examples 3 and 4], the reader may also construct interesting examples of pseudo-injective modules $M$ over a noncommutative ring in a manner somewhat similar to Example 3.

\section{REFERENCES}

1. L. Fuchs, Torsion preradicals and ascending Loewy series of modules, J. 
Reine Angew. Math. 239/240 (1969), 169-179. MR 44 \#2783.

2. R. R. Hallett, Injective modules and their generalizations, Ph.D. Thesis, Univ. of British Columbia, Vancouver, B.C., 1971.

3. S. K. Jain, Talk at Special Session on Ring Theory, Amer. Math. Soc. Meeting 711, San Francisco, 1974.

4. S. K. Jain and S. Singh, Quasi-injective and pseudo-injective modules, preprint, 1974.

5. R. E. Johnson and E. Wong, Quasi-injective modules and irreducible rings, J. London Math. Soc. 36 (1961), 260-268.

6. S. Singh and S. K. Jain, On pseudo-injective modules and self-pseudo-injective rings, J. Math. Sci. 2 (1967), 23-31. MR 36 \#2649.

7. S. Singh and $\mathrm{K}$. Wasan, Pseudo-injective modules over commutative rings, J. Indian Math. Soc. 34 (1970), 61-65. MR 44 \#6676.

DEPARTMENT OF MATHEMATICS, UNIVERSITY OF FLORIDA, GAINESVILLE, FLORIDA 32611 